\title{
Phytosociological study of weeds of wheat crop under edaphic variation: A case study from district Swabi, Khyber Pakhtunkhwa, Pakistan
}

\author{
Maqsood Anwar", Naveed Akhtar and Shah Khalid \\ Department of Botany, Islamia College Peshawar-Pakistan \\ *Corresponding author's e-mail: maqsoodanwarbot@yahoo.com \\ Citation \\ Maqsood Anwar, Naveed Akhtar and Shah Khalid. Phytosociological study of weeds of wheat crop under edaphic \\ variation: A case study from district Swabi, Khyber Pakhtunkhwa, Pakistan. Pure and Applied Biology. Vol. 9, Issue \\ 1, pp640-649. http://dx.doi.org/10.19045/bspab.2020.90070
}

\begin{tabular}{llll}
\hline \hline Received: 18/09/2019 & Revised: 01/12/2019 & Accepted: 03/12/2019 & Online First: 20/12/2019 \\
\hline
\end{tabular}

\section{Abstract}

The present study explains phytosociological attributes and communities structure of weeds of wheat crop under edaphic variation. This study was conducted in tehsil Razar district Swabi, Khyber Pakhtunkhwa, Pakistan during April 2016. A total of 200 quadrats having size $\left(1 \times 1 \mathrm{~m}^{2}\right)$ were established in 20 randomly selected wheat fields. Analytical quantitative characters i.e. density, frequency and cover were calculated for each weed species. Twenty weed communities were recognized on the basis of highest importance value (HIV) of three leading species. For each community, importance values (IV) of weed species were added to obtained total important value (TIV) of three dominant species and the remaining associated species. Sorensen's index was used to study similarity between weed communities. Higher similarity $(76.1 \%)$ was recorded between Veronica-Papaver-Phalaris community and Trifolium-Cynodon-Melilotus community while lower similarity (16.6\%) was recorded between Anagallis-Emex-Phalaris community and Veronica-Cerastium-Anagallis community. Physico-chemical analysis of soil showed that texture ranged from sandy-loam to loam, sandy clay-loam, slit-loam and loamy-sand or sand. Soil $\mathrm{pH}$ ranged from 5.8 to 8.1. Electrical conductivity (EC) ranged from $0.06 \mathrm{dsm}^{-1}$ to $0.23 \mathrm{dsm}^{-1}$. Organic matter $(\mathrm{OM})$ varied from $0.15 \%$ to $1.94 \%$. While Calcium carbonates $\left(\mathrm{CaCO}_{3}\right)$ ranged from $5.5 \%$ to $23.5 \%$. It was concluded that soil texture, $\mathrm{pH}$, electrical conductivity, Calcium carbonates and organic matter were strong edaphic variables that gives rise to diverse weed species composition, richness and distribution. This research will provide baseline knowledge about the weed ecology and it will be helpful in future studies.

Keywords: Phytosociology; Razar; Similarity index; Soil analysis; Weeds; Wheat

\section{Introduction}

Phytosociology is the branch of botany which deals with the structure, composition and development of plant communities and the relationships between the species within them. The term "Phytosociology" was first coined by "Jozef Paczoski" in 1896 [1]. According to many authors phytosociology is the branch of plant science which deals with the study of vegetation as such, with its 
floristic structure, development and distribution. Phytosociology is a sub branch of plant ecology which deals with the cooccurrence of plant species in communities [2].

Weeds are unwanted plants growing in cultivated as well as in domesticated areas. Weeds are adapted to various edaphic and climatic conditions. These are distributed everywhere and no crop is free of weeds. Plant species of family Asteraceae, Brassicaceae, Fabaceae and Poaceae constitutes major weed flora in cultivated crops. [3] reported that there are almost 30,000 weed plant species in world, of them, more than 50 causing considerable damage to agricultural crops. Weeds are strong competitors and hidden enemies of crops due to their vigorous growth and compete with crops mainly for nutrients, space, water and light and also decreases quantity as well as quality of crops [4]. Seedlings of weed plants grow vigorously in crop fields and spread in an area within a short time because their seeds germinate earlier to crops. Weeds develop a mutualistic association with insect pollinators and successfully invade new area and affecting various ecosystems [5, 6]. [7] stated that the invasive weed species change the structure and composition of native plants and as a result creates pressure on food chain and food web. Some weed plants secretes certain allelochemicals which affect the growth and germination of agricultural crops [8]. The present study aimed to phytosociological attributes and communities structure of weeds of wheat crop under edaphic variation at tehsil Razar district Swabi, Khyber Pakhtunkhwa, Pakistan.

\section{Materials and methods \\ Study area}

Razar is recently announced tehsil in district Swabi Khyber Pakhtunkhwa, Pakistan. It is lies from $34^{\circ} 6^{\prime} 0^{\prime \prime}$ to $34^{\circ} 23^{\prime} 0^{\prime \prime}$ North latitudes and $72^{\circ} 12^{\prime} 0^{\prime \prime}$ to $72^{\circ} 27^{\prime} 0^{\prime \prime}$ East longitudes. The total area of tehsil Razar is approximately $385 \mathrm{Km}^{2}$ and its population is 583,936 as per census of 2017 . The soil of the area is fertile and used for general cropping. It shows great variation in texture and color. Area has harsh climate. Summer is very hot. July and August records quite high temperature. Dust storms are very frequent at night during May and June. A rapid fall in temperature observed from October onwards. Winter is very cold. January is coldest month. Maximum rainfall is received in monsoon (July and August) during which the weather become humid and hot. Maximum humidity has been recorded in December [9].

\section{Phytosociological study}

A total of 20 wheat fields were selected randomly in the study area. At each field, 10 quadrats (square shaped) having size of $\left(1 \times 1 \mathrm{~m}^{2}\right)$ were placed systematically [10]. The number of weed species were counted in each quadrat and analytical quantitative characters i.e. density, frequency and cover were determined for each weed species. The relative value $(\mathrm{RV})$ of each parameter for weed species were added to get the importance value (IV) $[10,11]$. Weed communities were named after the three leading species having the highest importance value (HIV). For each community, importance values (IV) of weed species were added to obtained total important value (TIV) of three dominant species and the remaining associated species. Index of similarity was calculated according to Sorensen's index [12].

\section{Soil analysis}

Soil samples were collected from 0-20 cm depth at all 20 selected fields. Soil texture was determined by Hydrometer method [13]. Texture classes were determined using texture triangle [14]. Soil $\mathrm{pH}$ was determined with the help of $\mathrm{pH}$ meter $[15,16]$. Electrical conductivity (EC) was determined with the help of electrical conductivity meter [16]. Soil organic matter was determined after 
[17]. Calcium carbonate was determined by acid neutralization method [18].

\section{Results and discussion}

\section{Phytosociology/Communities structure}

Twenty weed communities were named and recognized on the basis of highest importance value (HIV) of three leading species. For each community, importance values (IV) of weed species were added to obtained total important value (TIV) of three dominant species and the remaining associated species $(\mathrm{TIV}=3$ dominant species; remaining associated species). These communities were 1) Cynodon-Veronica-Medicago community $(\mathrm{TIV}=126.42 ; 172.86), \quad 2)$ Stellaria-PoaAnagallis community (TIV=97.12;202.37), 3) Coronopus-Anagallis-Polygonum community (TIV=66.23;233.08), 4) Veronica-Papaver-Phalaris community (TIV=120.75;178.85), 5) Poa-AnagallisAvena community (TIV=90.2;209.69), 6) Galium-Anagallis-Phalaris community (TIV=83.6;216.07), 7) Medicago-CynodonPolygonum community (TIV=101.76;197.76), 8) CynodonAnagallis-Polygonum community (TIV=60.83;238.64), 9) Cynodon-MedicagoCerastium community

(TIV=101.38;198.83), 10) Poa-MedicagoAnagallis community (TIV=134.34;165.34), 11) Trifolium- Cynodon-Melilotus community $\quad(\mathrm{TIV}=134.58 ; 149.82), \quad 12)$ Chenopodium-Emex-Phalaris community $(\mathrm{TIV}=117.54 ; 182.11), 13)$ Anagallis-EmexPhalaris community (TIV=131.81;149.98), 14) Medicago-Anagallis-Coronopus community $\quad(\mathrm{TIV}=132.17 ; 177.41), \quad 15)$ Veronica-Cerastium-Anagallis community $(\mathrm{TIV}=142.89 ; 156.92), \quad 16) \quad$ MedicagoVeronica-Parthenium community (TIV=134.4;180.27), 17) MedicagoFumaria-Phalaris community $(\mathrm{TIV}=132.85 ; 166.83), \quad 18) \quad$ Anagallis-
Medicago-Fumaria

(TIV=68.43;230.59),

community Medicago-Rananculus Melilotuscommunity (TIV=121.47;177.31) and 20) PolypogonRananculus-Veronica community (TIV=158.11;141.44) (Table 1; Figure 1). While studying weeds of wheat, [19-23] named and recognized weed communities on the basis of highest importance values (HIV) of three dominant species in wheat crop at different parts of Pakistan. Based on highest total importance values (TIV > 100), the dominant weed species of these communities were Medicago polymorpha, Anagallis arvensis, Cynodon dactylon, Veronica persica. Poa annua, Phalaris minor, Cerastium dichotomum, Stellaria media, Melilotus indica, Rananculus muricatus, Avena sativa, Coronopus didymus, Polygonum plebeium, Fumaria indica, Polypogon monspeliensis, Emex spinosa and Parthenium hysterophorus. The total and average importance values (TIV; AIV) of these weed species in communities were (545.39; 27.27), (463.45; 23.17), (389.27; 19.46), (346.05; 17.3), (288.21; 14.41), (260.89; 13), (260.71; 13), (232.72; 11.63), (212.54; 10.62), (202.29; 10.11), (161.82;8), (149.56; 7.48), (147; 7.35), (146; 7.68), (144.15; 7.21), (127.44; 6.37) and (114.71; 5.73) respectively (Figure 2). The present findings support the results of [24-30] who listed Avena sativa, Anagallis arvensis, Coronopus didymus, Chenopodium album, Cynodon dactylon, Emex spinosa, Fumaria indica, Melilotus indica, Medicago polymorpha, Phalaris minor, Polypogon monspeliensis, Polygonum plebeium, Parthenium hysterophorus, Poa annua, Rananculus muricatus, Stellaria media and Veronica persica as dominant weed species based on highest important values (HIV). 
Table 1. Total number of species and total importance values of weed communities of wheat crop at tehsil Razar, district Swabi, Pakistan.

\begin{tabular}{|c|c|c|c|c|}
\hline S. & Weed Communities & Total species in & \multicolumn{2}{|c|}{ Total Importance Values (TIV) } \\
\cline { 4 - 5 } No. & the community & 3 dominant species & Associated species \\
\hline $\mathbf{1}$ & Cynodon-Veronica-Medicago community & 21 & 126.42 & 172.86 \\
\hline $\mathbf{2}$ & Stellaria-Poa-Anagallis community & 32 & 97.12 & 202.37 \\
\hline $\mathbf{3}$ & Coronopus-Anagallis-Polygonum community & 34 & 66.23 & 233.08 \\
\hline $\mathbf{4}$ & Veronica-Papaver-Phalaris community & 22 & 120.75 & 178.85 \\
\hline $\mathbf{5}$ & Poa-Anagallis-Avena community & 26 & 90.2 & 209.69 \\
\hline $\mathbf{6}$ & Galium-Anagallis-Phalaris community & 23 & 83.6 & 216.07 \\
\hline $\mathbf{7}$ & Medicago-Cynodon-Polygonum community & 32 & 101.76 & 197.76 \\
\hline $\mathbf{8}$ & Cynodon-Anagallis-Polygonum community & 41 & 60.83 & 238.64 \\
\hline $\mathbf{9}$ & Cynodon-Medicago-Cerastium community & 25 & 101.38 & 198.83 \\
\hline $\mathbf{1 0}$ & Poa-Medicago-Anagallis community & 21 & 134.34 & 165.34 \\
\hline $\mathbf{1 1}$ & Trifolium-Cynodon-Melilotus community & 20 & 134.58 & 149.82 \\
\hline $\mathbf{1 2}$ & Chenopodium-Emex-Phalaris community & 22 & 117.54 & 149.11 \\
\hline $\mathbf{1 3}$ & Anagallis-Emex-Phalaris community & 21 & 131.81 & 177.41 \\
\hline $\mathbf{1 4}$ & Medicago-Anagallis-Coronopus community & 26 & 132.17 & 156.92 \\
\hline $\mathbf{1 5}$ & Veronica-Cerastium-Anagallis community & 15 & 142.89 & 180.27 \\
\hline $\mathbf{1 6}$ & Medicago-Veronica-Parthenium community & 15 & 134.40 & 166.83 \\
\hline $\mathbf{1 7}$ & Medicago-Fumaria-Phalaris community & 21 & 132.85 & 230.59 \\
\hline $\mathbf{1 8}$ & Anagallis-Medicago-Fumaria community & 27 & 68.43 & 177.31 \\
\hline $\mathbf{1 9}$ & Melilotus-Medicago-Rananculus community & 21 & 121.47 & 141.44 \\
\hline $\mathbf{2 0}$ & Polypogon-Rananculis-Veronica community & 25 & 158.11 & \\
\hline & & & & \\
\hline
\end{tabular}

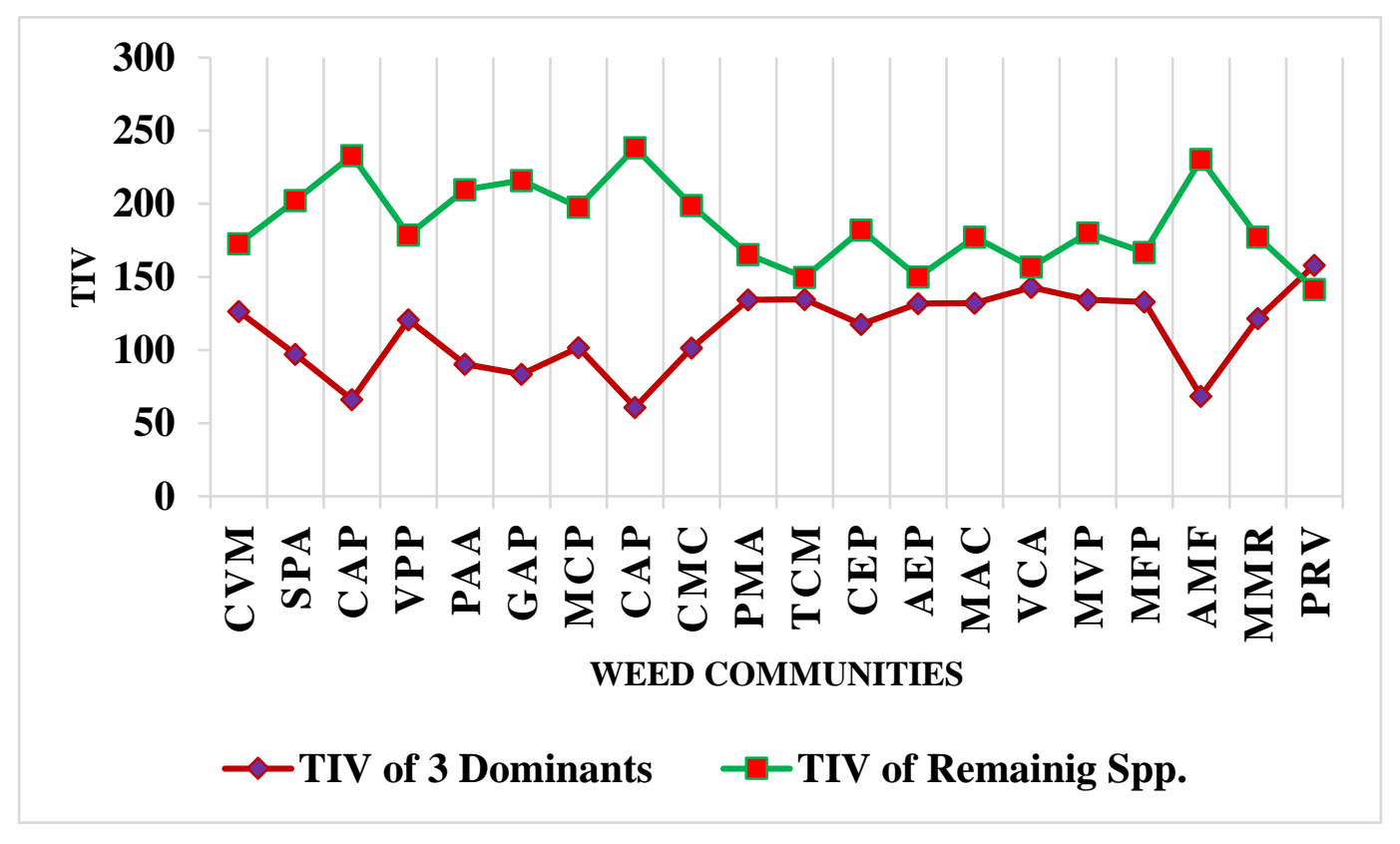

Figure 1. Total Important Values of three dominant and associated species in each weed community. For abbreviations of communities' names refers to Table 1. 


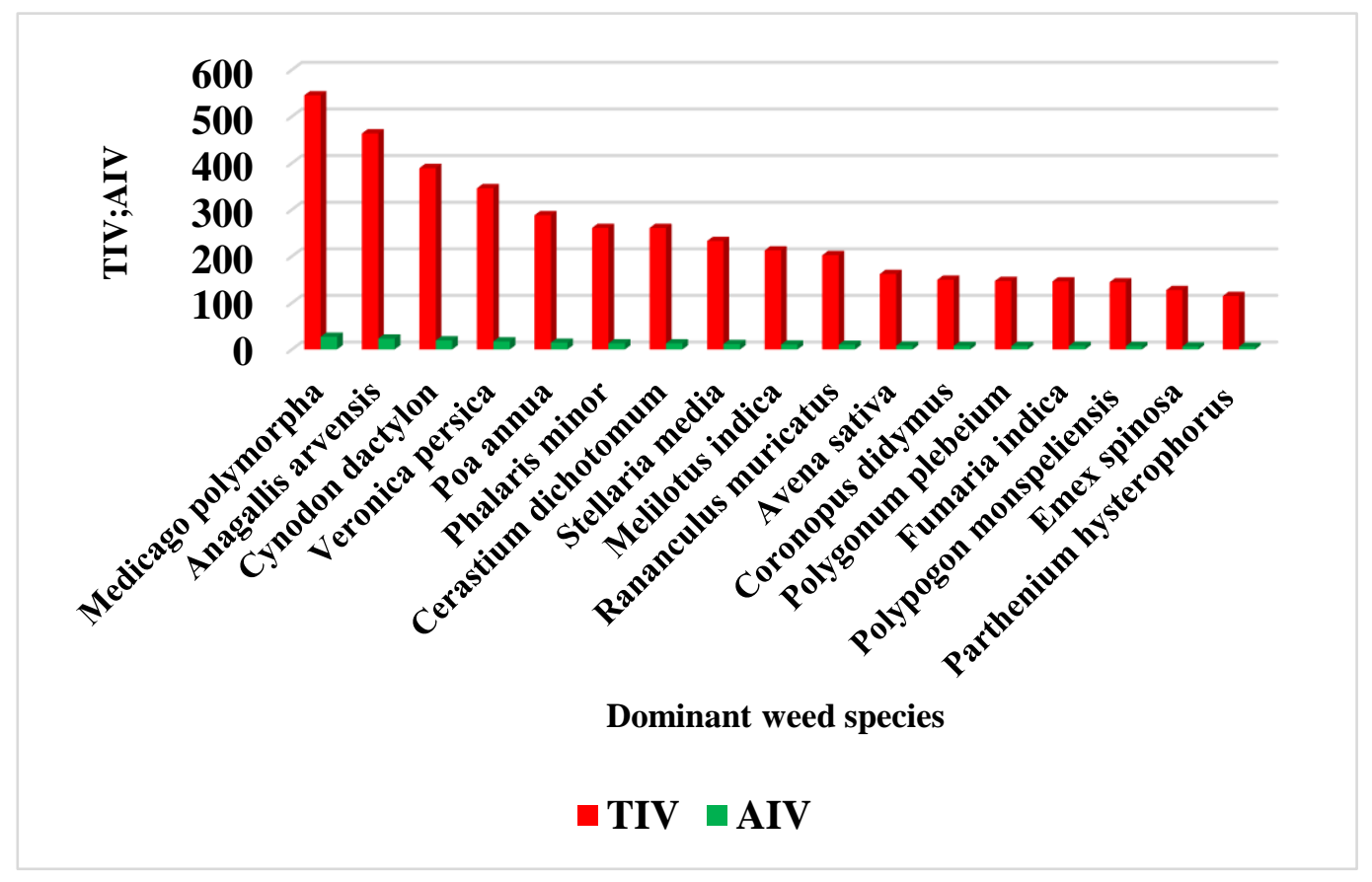

Figure 2. Total Important Values and Average Important Values of dominant weed species.

\section{Similarity index}

Higher similarity (76.1\%) was observed between Veronica-Papaver-Phalaris community and Trifolium-CynodonMelilotus community followed by $(75.5 \%)$ in Cynodon-Medicago-Cerastium community and Trifolium-Cynodon-Melilotus community, Trifolium-Cynodon-Melilotus community and Veronica-CerastiumAnagallis community had similarity index (74.2\%), Trifolium-Cynodon-Melilotus community and Medicago-VeronicaParthenium community (74.2\%), PoaMedicago-Anagallis community and Cynodon-Medicago-Cerastium community (73.9\%),

Galium-Anagallis-Phalaris community and Poa-Anagallis-Avena community (73.4\%), Poa-MedicagoAnagallis community and TrifoliumCynodon-Melilotus community (73.1\%), Medicago-Veronica-Parthenium community and Poa-Medicago-Anagallis community (72.2\%), Medicago-Cynodon-Polygonum community and Cynodon-AnagallisPolygonum (71.2\%), Medicago-Fumaria-
Phalaris community and AnagallisMedicago-Fumaria community (70.8\%), Poa-Anagallis-Avena community and Cynodon-Medicago-Cerastium community (70.5\%), Stellaria-Poa-Anagallis community and Veronica-Papaver-Phalaris community (70.3\%), Veronica-Papaver-Phalaris community and Veronica-CerastiumAnagallis community (70.2\%) and CynodonMedicago-Cerastium community and Medicago-Veronica-Parthenium community (70.0\%). Other communities showed similarity less than $70.0 \%$. While lower similarity less than $27.2 \%$ was observed between Anagallis-Emex-Phalaris community and Medicago-VeronicaParthenium community (22.2\%) followed by (21.7\%) in Anagallis-Emex-Phalaris community and Polypogon-RananculusVeronica community and (16.6\%) in Anagallis-Emex-Phalaris community and Veronica-Cerastium-Anagallis community (Table 2). Index of Similarity is used for comparing different plant communities in specific area. High similarity between plant 
communities is due to similarity in soil textures, $\mathrm{pH}$, electrical conductivity, nutrients and organic matter. Least similarity between plant communities is due to variation in climate and soil conditions. [31] studied index of similarity between different weed communities of wheat at district Swat, Pakistan. Similarly, [23] determined similarity index for weed communities of wheat at village Lahor, district Swabi, Pakistan, which strongly support the present findings.

\section{Soil analysis}

Physico-chemical analysis of soil showed that the texture ranged from sandy-loam to loam, sandy clay-loam, slit-loam and loamysand or sand. Soil $\mathrm{pH}$ ranged from 5.8 for Poa-Anagallis-Avena community to 8.1 for Cynodon-Anagallis-Polygonum community. Electrical conductivity ranged from 0.06 $\mathrm{dsm}^{-1}$ for Poa-Medicago-Anagallis community to $0.23 \mathrm{dsm}^{-1}$ for MelilotusMedicago-Rananculus community. Organic matter varied from $0.15 \%$ for PolypogonRananculus-Veronica community to $1.94 \%$ for Medicago-Veronica-Parthenium community. While $\mathrm{CaCO}_{3}$ ranged from $5.5 \%$ for Cynodon-Medicago-Cerastium community to $23.5 \%$ for Anagallis-EmexPhalaris community (Table 3). Soil is main abiotic ecological factor that play important role in the distribution of plant communities in particular area. Soil texture, electrical conductivity, $\mathrm{pH}$, organic matter and carbonates play key role in weed species diversity and distribution. [26] stated that texture, electrical conductivity, $\mathrm{pH}$, organic matter and carbonates play an important role in weed species diversity. [32] reported that soil organic matter is main soil fertility factor that affects the distribution of plant diversity. Furthermore, [33] reported that soil texture control infiltration rate, water holding capacity, availability of moisture and plant nutrition. Soil texture, organic carbon and salinity are main three factors for weed species richness and composition [34-36]. Generally, the soil of the study area is basic and rarely acidic. Compare to [25] who found same type of soil $\mathrm{pH}$ ranges 6.5-7.5 from Doon Valley India while studying weed communities of wheat fields, which strongly support our findings. [20] analyzed three soil series for texture, electrical conductivity, $\mathrm{pH}$, and organic matter. Similarly, [27] studied phytosociology of weeds of wheat crop under the edaphic variations i.e. Soil texture, electrical conductivity, $\mathrm{pH}$ and organic matter. 
Table 2. Similarity between weed communities of wheat crop at tehsil Razar, district Swabi, Pakistan.

\begin{tabular}{|c|c|c|c|c|c|c|c|c|c|c|c|c|c|c|c|c|c|c|c|}
\hline Weed Communities & & & & & & & & & & & & & & & & & & & \\
\hline Cynodon-Veronica-Medicago community & $\mathrm{X}$ & & & & & & & & & & & & & & & & & & \\
\hline Stellaria-Poa-Anagallis community & 56.6 & $\mathrm{X}$ & & & & & & & & & & & & & & & & & \\
\hline Coronopus-Anagallis-Polygonum community & 58.1 & 66.6 & $\mathrm{X}$ & & & & & & & & & & & & & & & & \\
\hline Veronica-Papaver-Phalaris community & 65.1 & 70.3 & 67.8 & $\mathrm{X}$ & & & & & & & & & & & & & & & \\
\hline Poa-Anagallis-Avena community & 63.8 & 58.6 & 56.6 & 62.5 & $\mathrm{X}$ & & & & & & & & & & & & & & \\
\hline Galium-Anagallis-Phalaris community & 50.0 & 61.8 & 52.6 & 62.2 & 73.4 & $\mathrm{X}$ & & & & & & & & & & & & & \\
\hline Medicago-Cynodon-Polygonum community & 56.6 & 59.3 & 66.6 & 48.1 & 58.6 & 54.5 & $\mathrm{X}$ & & & & & & & & & & & & \\
\hline Cynodon-Anagallis-Polygonum community & 58.0 & 57.5 & 64.0 & 50.7 & 50.7 & 56.2 & 71.2 & $\mathrm{X}$ & & & & & & & & & & & \\
\hline Cynodon-Medicago-Cerastium community & 56.5 & 66.6 & 64.4 & 68.0 & 70.5 & 66.6 & 63.1 & 54.5 & $\mathrm{X}$ & & & & & & & & & & \\
\hline Poa-Medicago-Anagallis community & 52.3 & 52.8 & 50.9 & 65.1 & 59.5 & 54.5 & 49.0 & 48.3 & 73.9 & $\mathrm{X}$ & & & & & & & & & \\
\hline Trifolium-Cynodon-Melilotus community & 53.6 & 69.2 & 66.6 & 76.1 & 60.8 & 69.7 & 50.0 & 52.4 & 75.5 & 73.1 & $\mathrm{X}$ & & & & & & & & \\
\hline Chenopodium-Emex-Phalaris community & 60.4 & 55.5 & 64.2 & 50.0 & 41.6 & 53.3 & 55.5 & 57.1 & 51.0 & 46.5 & 57.1 & $\mathrm{X}$ & & & & & & & \\
\hline Anagallis-Emex-Phalaris community & 33.3 & 30.1 & 43.6 & 32.5 & 34.0 & 27.2 & 49.0 & 38.7 & 39.1 & 33.3 & 29.2 & 27.9 & $\mathrm{X}$ & & & & & & \\
\hline Medicago-Anagallis-Coronopus community & 42.5 & 37.9 & 53.3 & 41.6 & 46.1 & 44.8 & 58.6 & 53.7 & 58.8 & 51.0 & 47.8 & 45.8 & 55.3 & $\mathrm{X}$ & & & & & \\
\hline Veronica-Cerastium-Anagallis community & 55.5 & 46.8 & 48.9 & 70.2 & 58.5 & 57.8 & 38.3 & 42.8 & 60.0 & 66.6 & 74.2 & 54.0 & 16.6 & 39.0 & $\mathrm{X}$ & & & & \\
\hline Medicago-Veronica-Parthenium community & 50.0 & 46.8 & 44.9 & 59.4 & 58.5 & 63.1 & 51.0 & 42.8 & 70.0 & 72.2 & 74.2 & 48.6 & 22.2 & 48.7 & 66.6 & $\mathrm{X}$ & & & \\
\hline Medicago-Fumaria-Phalaris community & 57.1 & 64.1 & 54.5 & 69.7 & 51.0 & 54.5 & 49.0 & 51.6 & 65.2 & 57.1 & 68.2 & 41.8 & 42.8 & 46.8 & 55.5 & 50.0 & $\mathrm{X}$ & & \\
\hline Anagallis-Medicago-Fumaria community & 58.3 & 61.0 & 62.3 & 65.3 & 60.3 & 60.0 & 54.2 & 64.7 & 69.2 & 58.3 & 63.8 & 57.1 & 37.5 & 60.3 & 47.6 & 52.3 & 70.8 & $\mathrm{X}$ & \\
\hline Melilotus-Medicago-Rananculus community & 42.8 & 56.6 & 54.5 & 60.4 & 59.5 & 59.0 & 52.8 & 51.6 & 62.5 & 61.9 & 63.4 & 41.8 & 38.0 & 46.8 & 50.0 & 50.0 & 61.9 & 54.1 & $\mathrm{X}$ \\
\hline Polypogon-Rananculis-Veronica community & 30.4 & 42.1 & 40.6 & 34.0 & 47.0 & 50.0 & 42.1 & 36.3 & 60.0 & 43.4 & 57.7 & 38.3 & 21.7 & 31.3 & 30.0 & 40.0 & 39.1 & 46.1 & 52.1 \\
\hline
\end{tabular}


Table 3. Physico-chemical characteristics of soil of different weed communities of wheat crop at tehsil Razar, district Swabi, Pakistan.

\begin{tabular}{|c|c|c|c|c|c|c|c|c|c|}
\hline S. No. & Communities & pH (1:5) & EC (1:5) & O.M & $\mathrm{CaCO}_{3}$ & Sand & Silt & Clay & Textural Class \\
\hline ----- & ---------- & ------- & $\mathrm{dsm}^{-1}$ & \multicolumn{2}{|c|}{$\%$} & \multicolumn{3}{|c|}{$\%$} & ------------ \\
\hline 1 & CVM & 7.8 & 0.13 & 0.77 & 18.5 & 62.2 & 22.6 & 15.2 & Sandy loam \\
\hline 2 & SPA & 7.7 & 0.12 & 1.03 & 19.5 & 52.2 & 28.6 & 19.2 & Sandy loam \\
\hline 3 & CAP & 7.4 & 0.15 & 0.25 & 17.2 & 78.0 & 18.8 & 3.2 & Loamy sand \\
\hline 4 & VPP & 7.3 & 0.16 & 1.13 & 16.6 & 89.4 & 1.4 & 9.2 & Sandy \\
\hline 5 & PAA & 5.8 & 0.14 & 1.81 & 12.5 & 49.6 & 27.2 & 23.2 & Sandy clay-loam \\
\hline 6 & GAP & 7.6 & 0.20 & 1.03 & 16.8 & 74.0 & 8.8 & 17.2 & Sandy loam \\
\hline 7 & MCP & 7.8 & 0.11 & 1.29 & 22.2 & 68.8 & 14.0 & 17.2 & Sandy loam \\
\hline 8 & CAP & 8.1 & 0.08 & 1.03 & 18.7 & 82.4 & 4.2 & 13.4 & Loamy sand \\
\hline 9 & CMC & 6.9 & 0.13 & 0.51 & 5.5 & 33.4 & 54.8 & 11.8 & Silt loam \\
\hline 10 & PMA & 7.4 & 0.06 & 0.51 & 11.0 & 81.4 & 5.4 & 13.2 & Sandy loam \\
\hline 11 & TCM & 7.4 & 0.15 & 0.77 & 6.5 & 53.2 & 29.8 & 17.0 & Sandy loam \\
\hline 12 & CEP & 6.0 & 0.14 & 1.38 & 10.7 & 53.4 & 26.0 & 20.6 & Sandy clay-loam \\
\hline 13 & AEP & 7.2 & 0.16 & 0.25 & 23.5 & 41.6 & 49.8 & 8.6 & Loam \\
\hline 14 & MAC & 7.3 & 0.14 & 1.03 & 10.5 & 78.2 & 4.6 & 17.2 & Sandy loam \\
\hline 15 & $\mathrm{VCA}$ & 6.5 & 0.12 & 1.24 & 10.5 & 75.4 & 8.6 & 16.0 & Sandy loam \\
\hline 16 & MVP & 6.8 & 0.10 & 1.94 & 6.5 & 83.4 & 4.4 & 12.2 & Loamy sand \\
\hline 17 & MFP & 7.8 & 0.10 & 0.39 & 14.2 & 47.6 & 48.6 & 3.8 & Sandy loam \\
\hline 18 & AMF & 7.6 & 0.15 & 1.60 & 13.0 & 88.8 & 2.0 & 9.2 & Loamy sand \\
\hline 19 & MMR & 7.6 & 0.23 & 1.03 & 14.6 & 70.0 & 6.8 & 23.2 & Sandy clay-loam \\
\hline 20 & PRV & 7.9 & 0.17 & 0.15 & 16.0 & 43.4 & 52.0 & 4.6 & Silt loam \\
\hline
\end{tabular}

Note: For abbreviations of communities' names refers to Table $1 \& 2$.

\section{Conclusion}

It was concluded that soil texture, $\mathrm{pH}$, electrical conductivity (EC), Calcium carbonates and organic matter were the strong edaphic variables for diverse weed species composition, richness and distribution. The present finding suggests that a variety of weed plants infesting wheat crop in the study area that may cause losses to yield of wheat crop. For acquiring the better yield, it is necessary to take appropriate biological, chemical and mechanical measures for weed control.

\section{Authors' contributions}

Conceived and designed the experiments: $\mathrm{N}$ Akhtar, Performed the experiments: M Anwar, Analyzed the data: M Anwar, Contributed reagents/ materials/ analysis tools: S Khalid, Wrote the paper: M Anwar.

\section{References}

1. Rabatnov TA (1979). Phytocoenology. In: The Great Soviet Encyclopedia, $3^{\text {rd }}$ Ed.

2. Ewald J (2003). A critique for phytosociology. J Veg Sci 14 (2): 291296.

3. Mahmood TZ \& Niaz SA (1992). "Weeds in Cropped Land at Islamabad," NARC, Identification and control of weeds manual, national training course, Pakistan agriculture research council, Islamabad. pp. 79.

4. Dangwal LR, Singh A, Singh T, Sharma A \& Sharma C (2010). "Common weeds of Rabi (winter) crops of Tehsil Nowshera, District Rajouri (Jammu \& Kashmir), India." Pak J Weed Sci Res 16(1): 39-45. 
5. Jesse LC, Moloney KA \& Obrycki JJ (2006). Insect visitors of invasive plant Rosa multiflora (Rosaceae), In Lowa, USA. Weed Bio Manage 6: 235-340.

6. Morale CL \& Aizen MA (2006). Invasive mutualism and the structure of plant-pollinators interaction in temperate forest of northwest Patagonia, Argentina. J Ecol 94: 171180.

7. Pysek P \& Richardson DM (2007). Traits associated with invasiveness in alien plants: where do we stand? In: Nentwig W (ed) Biological invasions. Springer, Berlin. pp. 97-126.

8. Oudhia P \& Tripathi RS (1998). Allelopathic effects of Parthenium hysterophorus L. on kodo, mustard and problematic weeds. $1^{\text {st }}$ Int. Conf. Parthenium management UAS, Dhaward, India. 6-8 Oct. 1997: 136-139.

9. Anwar M, Khan WM, Khan MS, Murad W \& Ali S (2015). Taxonomic study of Family Papilionaceae of District Swabi, Khyber Pakhtunkhwa, Pakistan. Pure Appl Bio 4(1): 125-128.

10. Hussain F (1989). Field and Laboratory Manual for Plant Ecology. University Grants Commission, Islamabad.

11. Ahmad M \& Shaukat SS (2012). A text book of Vegetation Ecology. Abrar Sons Karachi, Pakistan. pp. 29-76.

12. Sorensen TA (1948). A method of establishing groups of equal amplitude in plant sociology based on similarity of species content, and its application to analyses of the vegetation on Danish commons. Biol Skr K Danske Vidensk Selsk 5(4): 1-34.

13. Bouyoucos GJ (1936). Directions for making mechanical analysis of soils by the hydrometer method. Soil Sci 42: 225228.

14. Brady NC (1990). The Nature and Properties of Soils. 10th Ed. Macmillan Publishing Co. New York. $621 \mathrm{p}$
15. Jackson ML (1962). Soil chemical Analysis. Constable \& Co., Ltd., London. pp. 406-407.

16. Rhoades JD, Miyamoto $S$ \& Westerman RL (1990). Testing soils for salinity and sodicity. Soil Test. Plant Anal 299-336.

17. Black CA (1965). Methods of Soil Analysis. Agron Inc. Madison Wisconsin, USA.

18. Richard LA (1954). Diagnoses and improvement of saline and alkali soils. Agriculture Hand Book 60: USDA, USA.

19. Qureshi R \& Bhatti GR (2001). Determination of weed communities of wheat (Triticum aestivum L.) fields of district Sukkur. Pak J Bot 33(1): 109115.

20. Jakhar GS, Mahar AQ, Abro SA \& Qureshi R (2005). Weed communities of wheat crop under diverse Edaphography of District Khairpur. Pak J Bot 37(3): 709-714.

21. Qureshi R, Waheed A \& Arshad M (2009). Weed communities of wheat crop in district Toba Tek Singh, Pakistan. Pak J Bot 41(1): 239-245.

22. Waheed A, Qureshi R, Jakhar GS \& Tareen H (2009). Weed community dynamics in wheat crop of district Rahim Yar Khan, Pakistan. Pak J Bot 41: 247254.

23. Sher Z, Hussain F, Badshah L \& Wahab M (2011). Floristic composition, communities and ecological characteristics of weeds of wheat fields of Lahor, District Swabi, Pakistan. Pak J Bot 43(6): 2817-2820.

24. Kazi BR, Buriro AH, Kubar RA \& Jagirani AW (2007). Weed spectrum frequency and density in wheat (Triticum aestivum L.) under Tandojam conditions. Pak J Weed Sci Res 13(3-4): 241-246.

25. Gupta A, Joshi SP \& Manhas RK (2008). Multivariate analysis of diversity and 
composition of weed communities of wheat fields in Doon Valley, India. Trop Eco 49(2): 103-112.

26. Gomaa NH (2012). Composition and diversity of weed communities in AlJouf Province, Northern Saudi Arabia. Saudi J Biol Sci 19: 369-376.

27. Malik ZH, Amjad MS, Rafique S \& Malik NZ (2013). Phytosociology of some weeds of wheat communities around Kotli fields, Western Himalaya. J Eco Nat Envi 5(11): 340-345.

28. Marwat SK, Usman K, Khan NU, Khan MU, Khan EA, Khan MA \& Rehman AU (2013). Weeds of Wheat Crop and Their Control Strategies in Dera Ismail Khan District, Khyber Pakhtunkhwa, Pakistan. Amer J Plant Sci 4: 66-76.

29. Inayat $\mathrm{N}$, Asadullah \& Rashid A (2014). Floristic composition and ecological prevalence of the weed species growing in wheat and sugarcane fields of district Charsadda, Khyber Pakhtunkhwa, Pakistan. PakJ Weed Sci Res 20(3): 405415.

30. Khobragade DP \& Sathawane KN (2014). Weed diversity in Rabi wheat crop of Bhandara District (MS), India, Inter J Life Sci 2(Special Issue): 128131.
31. Akhtar N \& Hussain F (2007). Weeds of wheat fields of village Qambar, District Swat, Pakistan. Pak J Pl Sci 13(1): 3135.

32. Zhang K, Dang H, Tan SH, Wang ZH \& Zhang Q (2010). Vegetation community and soil characteristics of abandoned agricultural land and pine plantation in the Qinling Mountains, China. Forest Ecol Manage 259: 2036-2047.

33. Sperry JS \& Hacke UG (2002). Desert shrub water relations with respect to soil characteristics and plant functional type. Funct Ecol 16: 367-378.

34. Fried G, Norton LR \& Reboud X (2008). Environmental and management factors determining weed species composition and diversity in France. Agric Ecosyst Environ 128: 68-76.

35. Andreasen C \& Skovgaard IM (2009). Crop and soil factors of importance for the distribution of plant species on arable fields in Denmark. Agr Ecosyst Environ 133: 61-67.

36. Pinke G, Pal R \& Botta-Dukat Z (2010). Effects of environmental factors on weed species composition of cereal and stubble fields in western Hungary. Cent Eur J Biol 5(2): 283-292. 